\title{
Binafuxi granules in the treatment of common cold with heat syndrome based on traditional Uighur medicine: study protocol for a multicenter randomized controlled trial
}

\author{
Jie Min, Bin She, Xin Zhang, Bing Mao* and Yan Chen
}

\begin{abstract}
Background: The common cold is a highly prevalent illness with significant impact on society and health care. Common cold with heat syndrome (CCHS) is one of the most common types based on syndrome differentiation by traditional Uighur medicine (TUM), which is widely used in Central Asia. The study is designed to explore the efficacy, safety and optimal therapeutic dosage of Binafuxi granules in treating CCHS.

Methods: This is a multicenter, randomized, double-blind, placebo-controlled, phase II clinical trial. Participants ( $n=240$ ) will be enrolled from five centers across China and randomly assigned to the high-dose group, low-dose group or placebo control group in a 1:1:1 ratio. All eligible patients will receive test drugs twice daily for 3 days. The primary outcome is the time to fever relief. Secondary outcomes include the time to fever clearance, duration of primary symptoms and each symptom and change in TUM symptom score.
\end{abstract}

Discussion: This is the first placebo-controlled randomized clinical trial of a Uighur medicine in treating common cold. It will provide robust evidence on the efficacy and safety of Binafuxi granules in the treatment of CCHS.

Trial registration: Chinese Clinical Trial Registry, ChiCTR-IIR-17013379. Registered on 14 November 2017.

Keywords: Common cold, Traditional Uighur medicine, Heat syndrome, Binafuxi granules, Randomized controlled trial

\section{Background}

The common cold is a highly prevalent, upper respiratory tract, infectious disease, mainly caused by more than 200 viruses [1]. Varying among different viruses, the symptoms of the common cold often include sneezing, runny nose, nasal congestion, sore throat, coughing, fever and general malaise [2, 3]. The duration of these symptoms is generally $3-7$ days, but is occasionally up to several weeks [1]. Despite being generally mild, the common cold still has a significant impact on society and health care [4]. According to previous data, the common cold is the third most common diagnosis in

\footnotetext{
* Correspondence: maobing@medmail.com.cn

Pneumology Group, Department of Integrated Traditional and Western Medicine, West China Hospital of Sichuan University, 37 Guoxue Lane, Chengdu 610041, Sichuan Province, People's Republic of China
}

physician visits [5]. It even can be a trigger for severe or even fatal illness in individuals with chronic respiratory diseases or other underlying conditions [6].

So far, the treatment of common cold is aimed at symptom relief. Complementary and alternative medicine (CAM), primarily herbal and nutritive remedies, has become very popular recently [7]. Many clinical studies of CAM, such as on the use of echinacea, vitamin D, vitamin $\mathrm{C}$, probiotics, ginseng and Chinese herbal medicine, have demonstrated efficacy and safety in treating the common cold [8-14]. Traditional Uighur medicine (TUM) is the common traditional medicine used in Xinjiang Uygur Autonomous Region, China. With a history of more than 2500 years, it is based on the theory of ancient Uighur medicine, combining the theories of traditional Chinese medicine (TCM) and ancient Greek, Egyptian, Arabian

(c) The Author(s). 2019 Open Access This article is distributed under the terms of the Creative Commons Attribution 4.0 International License (http://creativecommons.org/licenses/by/4.0/), which permits unrestricted use, distribution, and 
and Indian medicine. The humorism, one of the most important TUM theories, is based on the four materials (fire, air, water and earth) and the four mizaj (temperaments) (hot, cold, moist and dry) [15], and the humorae theory indicates that the hilit (body fluid) is composed of kan (blood), belghem (phlegm), sapra (yellow bile), and savda (black bile) [16]. Based on humorism, the common cold, which is also called zukamu in TUM, can be divided into heat syndrome and cold syndrome. The common cold with heat syndrome (CCHS) is generally related to the imbalance status and abnormal change in kan and sapra in the four body fluids [17]. CCHS is primarily characterized by fever (body temperature $>38^{\circ} \mathrm{C}$ ), sore throat, nasal congestion, nasal discharge, cough and headache.

The Binafuxi is an Uighur herbal medicine prescription and derived from ancient books on Uighur medicine, including Mahzinul Murakkibat (Uighur Combination Medicine) and Karabadin Azam. It has been used for treating the CCHS for more than 300 years. The prescription is mainly composed of tianshanjincai (Viola tianshanica Maxim), heguotenggen (roots of Operculina turpethum), gancaojingao (extractum Glycyrrhizae), meiguihua (Flos rosae rugosae), sikamoniyazhi (Resina scammoniae) and alihong (Sclerotium fomitis officinalis). Binafuxi granules are based on the Binafuxi prescription and have been approved by the State Food and Drug Administration of China (SFDA) for a clinical trial (approval number 2014 L01342). Unpublished pharmacologic experiments have demonstrated that Binafuxi granules relieve fever, alleviate cough and sweating and reduce inflammation. The data showed that Binafuxi granules can significantly reduce endotoxin-induced fever in rabbits administered clinical equivalent doses $(0.52 \mathrm{~g} / \mathrm{kg})$ of Binafuxi granules. Additionally, there was no chronic toxicity found in rats administered different doses of Binafuxi granules (30, 15 and 7.5 times the normal dose) for 1 month except for some cases of diarrhea that occurred in the group receiving 30 times the normal dose.

Since ancient times, many patients have benefited from TUM treatment of the common cold. However, to our knowledge there have been no high-quality clinical studies of the use of TUM in treating the common cold. In conformity with the Drug Administration Law of the People's Republic of China and good clinical practice (GCP) issued by the SFDA, we designed this randomized clinical trial to evaluate the efficacy and safety of Binafuxi granules in patients with CCHS and to detect the optimal therapeutic dosage.

\section{Methods/design}

This trial is a multicenter, double-blind, placebo-controlled and randomized phase II clinical study, which has been authorized by the SFDA (approval number: 2014 L01342) and registered with the Chinese Clinical Trial Registry (ChiCTR-IIR-17013379). The study is financially supported by Xinjiang Yinduolan Uighur Medicine Co. Ltd., Xinjiang, China, which had or will have no role in the study design, study conduct, data management or decision to submit the study results. This trial will be conducted in five trial sites in China. A total of 240 patients will be enrolled and randomly allocated to the high-dose group, low-dose group or placebo

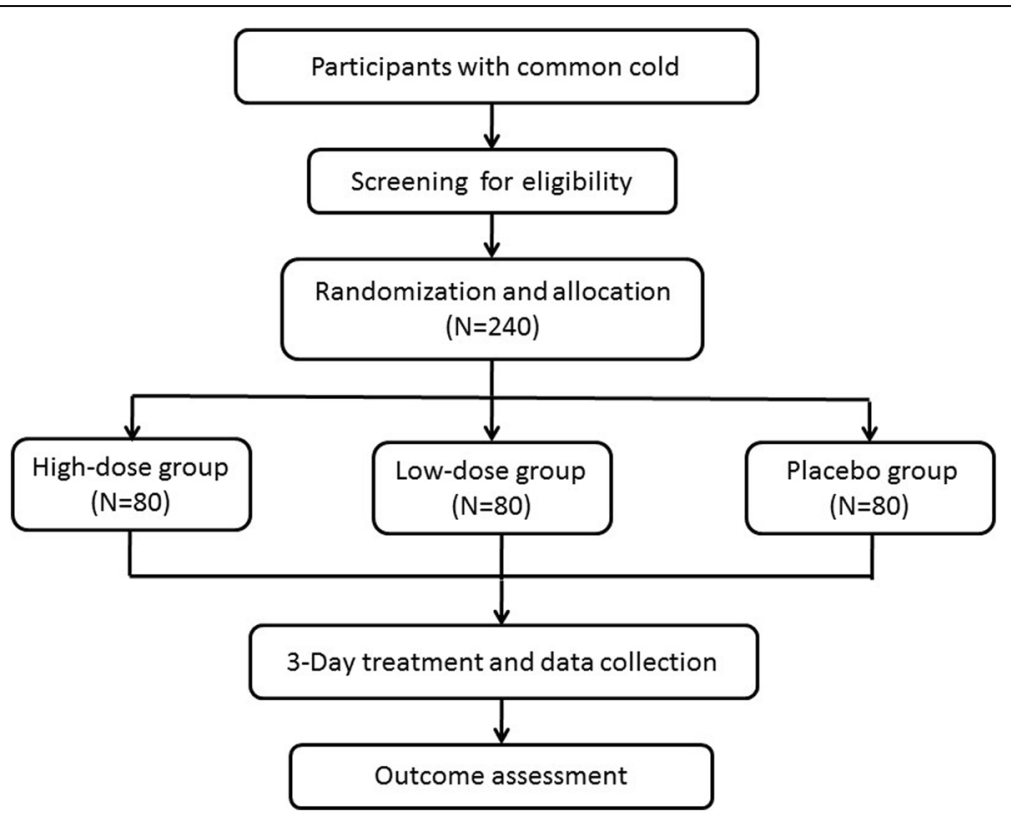

Fig. 1 Flowchart. We will recruit 240 patients who will randomly be allocated to one of three groups (high-dose group, $n=80 ;$ low-dose group, $n=80$; control group, $n=80$ ). After 3 days, we will analyze and assess the data. TUM, traditional Uighur medicine 
control group, in a 1:1:1 ratio. Binafuxi granules and placebo will be provided for 3 days to subjects in the three study groups, respectively. The study flow chart is shown in Fig. 1.

The trial protocol has been approved by the Ethics Committee of Clinical Trials and Biomedicine of West China Hospital of Sichuan University (IRB-2017-5). All eligible patients have to provide their signed informed consent prior to enrollment. The privacy of all subjects will be properly protected. The personal information on eligible patients will be concealed by identification codes and all records will be stored in a locked location.

\section{Recruitment}

By means of advertisements and recommendations, we recruit participants at the following five research sites across China: (1) West China Hospital of Sichuan University, (2) Shanghai Traditional Chinese Medicine Hospital, (3) Xinjiang Traditional Chinese Medicine Hospital, (4) Ruikang Hospital Affiliated to Guangxi University of traditional Chinese medicine and (5) Lishui People's Hospital. Each site will recruit patients in equal numbers.

Eligible participants in these five sites will be enrolled by the well-trained investigators. The investigators will talk with eligible patients about the study and obtain informed consent from them. The Principal Investigator is responsible for subject recruitment in each site.

\section{Inclusion criteria}

The inclusion criteria are as follows: (1) meet the diagnosis of common cold according to Western medicine; (2) meet the diagnosis of CCHS according to traditional Uighur medicine; (3) fever and body temperature between 38 and $39^{\circ} \mathrm{C}$; (4) symptom presenting within $24 \mathrm{~h}$; (5) age between 18 and 65 years and (6) voluntarily participate and provide signed informed consent.

\section{Exclusion criteria}

The exclusion criteria are as follows: (1) influenza, acute or chronic rhinitis, acute sinusitis, suppurative tonsillitis, pneumonia and tuberculosis; (2) white blood cell count $>11.0 \times 10^{9} / \mathrm{L}$ and/or neutrophil percentage $>75 \%$; (3) taking any medication to treat common cold; (4) liver function levels (alanine aminotransferase (ALT) and aspartate aminotransferase (AST)) 1.5 times higher than the upper limit of normal or abnormal serum creatinine; (5) serious primary cardiovascular, pulmonary, kidney, liver, neurological or hematological disease; (6) pregnant, lactating or planning to become pregnant; (7) allergic or possibility of being allergic to the ingredients in the study drug; (8) participating in or have participated in other clinical drug trials within the last 3 months and (9) identified by the investigator as inappropriate to participate in this study.

\section{Diagnostic criteria}

Diagnosis of the common cold in Western medicine is established according to the diagnostic criteria for the common cold of acute upper respiratory tract infection of Internal Medicine, $8^{\text {th }}$ Version (2013) [18]. The typical clinical symptoms of common cold include sneezing, nasal congestion, nasal discharge, fever and sore throat.

The TUM diagnosis of CCHS is based on the Clinical research guidelines for treatment of common cold with new Uyghur medicine (2017) [17]. The TUM diagnostic criteria for $\mathrm{CCHS}$ include primary symptoms, secondary symptoms, signs for the tongue and signs for the pulse. The primary symptoms include fever, nasal congestion, nasal discharge and sore throat. The secondary symptoms include cough, headache, thirst, sore limbs and sweating. The signs for the tongue are red tongue and thick yellow tongue coating. The signs for the pulse are hard and rapid pulse. To meet the diagnosis of CCHS, patients should have at least two of the primary symptoms in which fever is essential, and at least two of the secondary symptoms, as well as the TCM signs for the tongue and pulse.

\section{Interventions}

Binafuxi granules (batch Y170521) and placebo granules (batch Y170520) are manufactured by Xinjiang Yinduolan Uighur Medicine Co. Ltd., Xinjiang, China. The minimum effective dose of Binafuxi granules is $2.75 \mathrm{~g}$. The Chinese herbal medicine placebo consists of 20 -fold dilution of $2.75 \mathrm{~g}$ of Binafuxi granules and maltodextrin with the addition of artificial pigment and flavoring agents [19]. Patients in the placebo group will be administered placebo granules, which are almost identical to the Binafuxi granules in appearance, smell and taste. All test drugs are concealed in unified sealed packages and each package contains a 2.75-g dose. In each site, an independent drug administrator is responsible for dispensing, reclaiming, storing and recording of all test drugs. Patients in the high-dose group, low-dose group or placebo group will receive two packages of Binafuxi granules $(5.5 \mathrm{~g})$ twice daily, one package of Binafuxi granules $(2.75 \mathrm{~g})$ plus one placebo package $(2.75 \mathrm{~g})$ twice daily, or two placebo $(5.5 \mathrm{~g})$ twice daily, respectively. All test drugs will be dissolved in warm water and taken orally two times daily for 3 days. Throughout the trial, participants will be visited at baseline and on the fourth day post baseline (see Fig. 2). The study protocol complies with Standard Protocol Items: Recommendations for Interventional Trials (SPIRIT) (see Additional file 1). We will not regularly follow up all patients except in the case of an adverse event.

\section{Concomitant treatments and forbidden drugs}

During the study, if the body temperature of a patient rises above $39.0^{\circ} \mathrm{C}$ and/or the body temperature do not drop 


\begin{tabular}{|c|c|c|c|}
\hline & \multicolumn{3}{|c|}{ Study Period } \\
\hline & Enrollment & Allocation & Close-out \\
\hline Visits & Visit 1 & Visit 2 & Visit 3 \\
\hline Time point & Day 0 & Day 1 & Day 4 \\
\hline \multicolumn{4}{|l|}{ Basic medical record } \\
\hline Informed consent & $x$ & & \\
\hline Demographic information & $x$ & & \\
\hline Medical history & $x$ & & \\
\hline Comorbidity & $x$ & & \\
\hline Combined medication & $x$ & $x$ & $x$ \\
\hline Physical examination & $x$ & $x$ & $x$ \\
\hline Eligibility screen & $x$ & & \\
\hline \multicolumn{4}{|l|}{ Efficacy assessment } \\
\hline The time to fever relief & & & $\times$ \\
\hline The time to fever clearance & & & $x$ \\
\hline Duration of symptoms & & & $\times$ \\
\hline TUM symptom score & & $x$ & $x$ \\
\hline \multicolumn{4}{|l|}{ Safety assessment } \\
\hline Vital signs & $x$ & & $x$ \\
\hline Routine blood test & $x$ & & $x$ \\
\hline Urinalysis & $x$ & & $x$ \\
\hline Stool routine & $x$ & & $x$ \\
\hline Liver function test & $x$ & & $x$ \\
\hline Renal function test & $x$ & & $x$ \\
\hline Electrocardiogram & $x$ & & $x$ \\
\hline Chest $\mathrm{x}$-ray & $x$ & & $x$ \\
\hline Urine pregnancy test & $x$ & & $x$ \\
\hline Adverse event record & & & $x$ \\
\hline \multicolumn{4}{|l|}{ Other procedures } \\
\hline Drug distribution & & $x$ & \\
\hline Drug recycling and count & & & $x$ \\
\hline
\end{tabular}

Fig. 2 Study schedule and Standard protocol items: recommendation for interventional trials (SPIRIT) figure

significantly at $48 \mathrm{~h}$ post administration, paracetamol can be used to bring down fever following the physician's advice. Patients can continue their treatments for underlying conditions, such as diabetes mellitus or hypertension. Rescue treatment in the case of drug-related adverse events is also permitted in this study. Furthermore, considering the related impact on the study results, a patient with a drug-related adverse event may be withdrawn from this trial. All concomitant medications or treatments in the study must be recorded carefully in the case report form (CRF). Any other medication or therapy for common cold is not allowed during the study. 


\section{Randomization and blinding}

According to a stratified block randomization method, 240 patients are stratified by study center and randomly assigned to the high-dose group, low-dose group or placebo control group in a 1:1:1 ratio. The random sequence and the randomization list of each center will be generated by the independent specialist, using the PRCO PLAN function of the analysis system of SAS software (SAS, Cary, NC, USA). The independent drug administrator will assign numbered packs of study drugs to eligible patients in order by randomization list. The randomization sequence will be concealed in a lightproof sealed envelope, which will be kept by the leader and the Sponsor.

Participants, investigators and statisticians will be blind to the treatment allocation throughout the study. Test drugs and placebo will be identical in appearance, color and taste. All drugs are concealed in uniform packages with number labels. An emergency letter including the random sequence and assignment has been prepared in each center. In any emergency medical situation, such as serious adverse event or deteriorative condition, the unblinding process will be started after contacting the Sponsor and the primary investigator. The investigator should record the details of urgent unblinding and make sure the corresponding patient is excluded.

\section{Outcome measures}

\section{Primary outcomes}

The primary outcome of the study is the time to fever relief, which is defined as the time (number of hours) from the first dose of the test drug until the axillary temperature drops by at least $0.5^{\circ} \mathrm{C}$. All patients are required to record their axillary temperature in the subject diary every $1 \mathrm{~h}$ within the first $6 \mathrm{~h}$. Then, if the temperature is $\geq 37.3^{\circ} \mathrm{C}$, it should be recorded every $2-4$ $\mathrm{h}$, and if the temperature is $<37.3^{\circ} \mathrm{C}$, it should be recorded at 8 a.m. and 4 p.m. every day. If the temperature is $<37.2^{\circ} \mathrm{C}$ and does not rise for $24 \mathrm{~h}$, it does not need to be recorded. The investigator will check and collect the subject diary in which the data will be acquired and evaluated on the fourth day.

\section{Secondary outcomes}

Secondary outcomes include the time to fever clearance, duration of primary symptoms and each symptom and change in TUM symptom score.

Duration of all symptoms and each symptom The duration of all symptoms is defined as the number of hours from enrollment to the time that all symptoms completely disappear. Each patient is required to record any change in symptoms in the subject diary. The investigator will assess the duration of all symptoms and each symptom.
Change in TUM symptom score Currently, there is no grading evaluation standard for Uighur medicine. In this trial, the TUM symptom graded score system follows the Guidelines for clinical research on new drug of chinese medicine [20] (see Table 1). The primary symptoms are given a graded score (none $=0$, mild $=3$, moderate $=6$, severe $=9)$ and the secondary symptoms are given a graded score (none $=0$, mild $=1$, moderate $=2$, severe $=$ 3). TUM signs will also be assessed, but not scored. The total score of TUM symptoms (including primary and secondary symptoms) will be summed at baseline (symptom score before treatment) and the fourth day (symptom score after treatment). The sum of all symptom scores is the cumulative TUM symptom score. The change in cumulative TUM symptom score is assessed by the percentage of symptom score reduction (PSSR), which is calculated according to the following formula:

$$
\begin{aligned}
\text { PSSR } & =\left(\frac{\text { symptom score before treatment-symptom score after treatment }}{\text { symptom score before treatment }}\right) \\
& \times 100 \%
\end{aligned}
$$

Based on the therapeutic effect evaluation system, the improvement of TUM symptoms will be categorized into clinical recovery (PSSR $\geq 70 \%$ ) and invalid (PSSR $<70 \%$ ).

\section{Safety assessment}

Safety assessment, including physical examination and laboratory tests, will be performed at baseline and the fourth day. Physical examination includes vital signs (temperature, respiration, heart rate and blood pressure) and conventional examination (chest, abdomen and nervous system, etc.). Laboratory tests include routine blood test, urine analysis, stool test, measurement of blood electrolytes, liver function test (ALT, AST, alkaline phosphatase (ALP), total bilirubin (TBil) and gamma-glutamyl transpeptidase (GGT)) and renal function test (blood urea nitrogen (BUN), serum creatinine, microalbuminuria, urinary nag enzyme and serum cystatin C). In addition, before treatment, all patients are required to undergo a chest $\mathrm{x}$-ray and electrocardiogram, and female patients of reproductive age are required to take a urinary pregnancy test.

All adverse events (AEs) should be truly recorded in detail on the adverse event form (AEF) and followed up to completion. The patient experiencing an $\mathrm{AE}$ will be treated appropriately until recovery from it. A serious $\mathrm{AE}$ (SAE) is defined as an adverse event resulting in death, a life-threatening event, an illness requiring hospitalization or severe disability, and it should be recorded and reported to the Principal Investigator, SFDA, ethics committee and the Sponsor within $24 \mathrm{~h}$. 
Table 1 Symptom scoring system and TUM sign

\begin{tabular}{|c|c|c|c|c|}
\hline TUM Symptoms & Score & & & \\
\hline Primary & 0 & 3 & 6 & 9 \\
\hline Fever & None & $37.3^{\circ} \mathrm{C}-37.9^{\circ} \mathrm{C}$ & $38^{\circ} \mathrm{C}-38.5^{\circ} \mathrm{C}$ & $>38.5^{\circ} \mathrm{C}$ \\
\hline Nasal congestion & None & Mild & Moderate & Severe \\
\hline Nasal discharge & None & Occasionally & Sometimes & Frequently \\
\hline Sore throat & None & Dry throat with slight pain & $\begin{array}{l}\text { Significant with obvious } \\
\text { pain when swallowing }\end{array}$ & Sore and swollen throat with severe pain \\
\hline Secondary & 0 & 1 & 2 & 3 \\
\hline Cough & None & Occasionally in daytime & Occasionally day and night & Frequently day and night \\
\hline Headache & None & Slight and occasionally & Slight and lasting & Severe and affecting working \\
\hline Thirsty & None & Dry mouth & Moderate & Severe and drinking plenty of water \\
\hline Sweating & None & Mild & Moderate & Severe \\
\hline Sore limbs & None & Slight & Moderate & Severe \\
\hline Cumulative symptom score & \multicolumn{4}{|c|}{ Primary symptom scores + secondary symptom scores } \\
\hline TUM sign & \multicolumn{4}{|l|}{ Unscored } \\
\hline Tongue body & $\square \operatorname{Red}$ & \multicolumn{3}{|l|}{ 口Others: } \\
\hline Tongue coating & 口Thick and yellow & \multicolumn{3}{|l|}{ 口Others: } \\
\hline Pulse condition & 口Hard and rapid & \multicolumn{3}{|l|}{ 口Others: } \\
\hline
\end{tabular}

TUM traditional Uighur medicine

\section{Quality control}

As the monitoring center, Beijing QiHuang Clinical Drug Research Center is in charge of monitoring implementation of the trial protocol, the CRF data and the safety of the participants, as well as contacting and coordinating the various units. The center will assign the Clinical Research Associate (CRA) to regularly review the CRFs and monitor the row data every week. The CRA is blind to trial assignment. Monitoring results should be presented to the Principal Investigator in each site. The Principal Investigator will be responsible for the implementation of the trial, which should be following the standard operation procedure (SOP) for quality assurance and quality control. Any protocol modification should be documented with appropriate justification and approved by the Sponsor, primary investigator in each unit and statisticians. Then the final version should be presented to the Ethics Committee, and the SFDA if required.

\section{Sample size calculation}

Using "One-Way Analysis of Variance F-Tests using Effect Size" in PASS (version 15.0.5), we estimate the sample size with a significance level of 0.05. To our knowledge, no previous study has reported the effective size related to the time to fever relief in our target population (patients with common cold). Thus, we used $\eta 2$ as an alternative measure of effect size, calculated by:

$$
\sigma \mathrm{m}^{2} /\left(\sigma \mathrm{m}^{2}+\sigma^{2}\right)
$$

to estimate the sample size [21-23]. Generally, the effect size can be defined as follows: $\eta^{2}=0.0099 \approx 0.01$ is a small effect; $\eta^{2}=0.0588 \approx 0.06$ is a medium effect and $\eta^{2}=0.1379 \approx 0.14$ is a large effect [21-23]. In the study, we would like to determine the sample size required to detect a medium effect when the power is 0.90 . Therefore, a total sample of 204 subjects (68 in each group) would be required. Assuming an overall 15\% drop-out rate, 80 subjects should be recruited in each group. Finally, the total sample size is determined to be 240 patients.

\section{Data management}

All CRFs in triplicate will be reviewed by the investigators and CRA. The completed CRFs will be securely stored in a locked location and finally sent to two independent data administrators, who will responsible for data entry, inspection and management in a specified statistics center. Once the trial is completed, the Principal Investigator, Sponsor, data administrators and statisticians will perform a blind review to confirm the dataset. The final database will be locked and analyzed in line with the statistical analysis plan.

\section{Statistical analysis}

The independent statistician will conduct the data analysis using SAS 9.4 software (SAS, Cary, NC, USA), in 
accordance with the statistical analysis plan for this trial. The primary analysis set for efficacy is the full analysis set (FAS) with an intention-to-treat (ITT) principle, in which all patients treated with at least one dose of study drug and who have a clinical observation record should be involved. According to an ITT principle, the last-observation-carried-forward (LOCF) imputation method will be used for missing data. Patients who fulfil the protocol without any major deviations will be included in the per-protocol set (PPS). And all participants who receive at least one dose of the study drug and for whom safety data are available will be included the safety set (SS). Baseline characteristics of all subjects will be analyzed using descriptive statistics for continuous variables and categorical variables. Primary efficacy will be analyzed by comparing outcomes among three groups. Outcomes among three groups will be compared using analysis of variance (ANOVA) and the Bonferroni method. Covariates designated as potential confounders will include age, gender, body mass index (BMI) and duration of symptomatic illness prior to enrollment. Time to fever relief, time to fever clearance and duration of all symptoms will be estimated by the Kaplan-Meier technique and compared by the stratified log-rank test. The improvement of TUM symptoms will be estimated by descriptive analysis for number and proportion of patients achieving clinical recovery. Prespecified subgroup analysis and sensitivity analysis involving covariance analysis will be used to evaluate the primary outcomes according to age and gender. In the multicenter trial, the Cochran-Mantel-Haenszel $(\mathrm{CMH})$ test will be performed to analyze stratified variables. A two-sided $P$ value $<0.05$ is considered as statistically significant.

\section{Discussion}

To date, this study is the first randomized, double-blind, placebo-controlled trial to evaluate the efficacy and safety of Uighur medicine in treating the common cold. In Central Asia, TUM is one of the most common traditional medicines, which has benefited many people in China and the other countries. So far, most of TUM researches have focused on therapy of skin disease, urogenital disease, rheumatism, digestive system and respiratory system disease [24]. To our knowledge, there have been few reports of standardized clinical trials designed for use in TUM.

Binafuxi formula has been used in Xinjiang Uygur Autonomous Region of China for over 20 years and based on local clinical experience it has been proven effective in treating CCHS. Derived from five different regions in China, the results of our study will provide stronger evidence for wider use of Binafuxi granules. We designed this study to practice the methodology of syndrome differentiation for clinical research in TUM. According to the grading standard for TCM, we will first perform the
TUM symptom graded score system in this trial, and then verify if it is feasible. This will provide a scientific quantitative standard to estimate the efficacy of TUM in treating the CCHS. In the light of TUM theory on the CCHS and the characteristics of Binafuxi, the time to fever relief and the duration of fever will be primarily observed in this study [17]. The efficacy both of Western medicine and TUM will be evaluated, so as to provide more powerful evidence for TUM therapy for the common cold.

Nevertheless, there are two potential limitations in this study. During the screening period, we will not confirm viral titers to exclude potential patients with influenza since this would largely delay patient enrollment for the study and potentially miss the best time to intervene. However, all the experienced physicians involved for this study will carefully evaluate patients to avoid mistakenly recruiting unsuitable participants. In accordance with the exclusion criteria, we will strictly exclude any participants with body temperature $>39^{\circ} \mathrm{C}$ and severe respiratory symptoms. Patients with chills and/or other influenza-like symptoms will be excluded or advised to be tested for the influenza virus. Furthermore, in this study, we will not explore the mechanisms underlying the CCHS and potential pathways through which Binafuxi may work in treating the common cold, which may need further research.

In conclusion, this protocol is rigorously designed for exploring the efficacy and safety of Binafuxi granules in treating the common cold with heat syndrome. As far as we know, this study will be the first multicenter, double-blind, placebo-controlled and randomized clinical trial of a Uighur medicine in treating the common cold. In addition, it will provide robust evidence for assessment of the efficacy and safety of Binafuxi granules in treating the CCHS, and may help in selecting the optimal therapeutic dose for the next phase III clinical trial.

\section{Trial status}

The study is currently in the process of recruiting participants. Recruitment of participants commenced on 5 February 2018 and will be completed in early May 2019. The protocol version number is Z-BNFX-GR-II-2017YDL-01, dated 22 May 2017.

\section{Additional file}

Additional file 1: SPIRIT 2013 checklist: recommended items to address in a clinical trial protocol and related documents. (DOC $134 \mathrm{~kb}$ )

\section{Abbreviations}

AEs: Adverse events; ALP: Alkaline phosphatase; ALT: Alanine transferase; ANOVA: Analysis of variance; AST: Aspartate transferase; BMI: Body mass index; BUN: Blood urea nitrogen; CAM: Complementary and alternative medicine; CCHS: Common cold with heat syndrome; CRF: Case report form; ECG: Electrocardiogram; FAS: Full analysis set; GCP: Good clinical practice; GGT: Gamma-glutamyl transpeptidase; ITT: Intention to treat; LOCF: last observation carried forward; PPS: Per-protocol set; PSSR: Percentage of 
symptom score reduction; SFDA: State Food and Drug Administration; SOP: Standard operation procedure; SS: Safety set; TBil: Total bilirubin; TCM: Traditional Chinese medicine; TUM: Traditional Uighur medicine; USA: United States of America

\section{Acknowledgements}

Thanks to all participants in this trial and the staff of the five research centers.

\section{Funding}

The study is financially supported by Xinjiang Yinduolan Uighur Medicine Co. Ltd., Xinjiang, China. The Sponsor had and will have no role in the study design, study conduct, data management or the decision to publish this manuscript.

\section{Availability of data and materials}

The data will be available to other investigators.

\section{Authors' contributions}

$J M, B S, X Z, Y C$ and BM contributed to the design and development of the study protocol ethics, trial registration and the manuscript draft. XZ was responsible for statistical design. BM was the general supervisor for this research. BS and YC participated in the coordination of the trial and recruiting patients. All authors reviewed the content and approved the final version.

\section{Ethics approval and consent to participate}

The trial protocol has been approved by the Ethics Committee of Clinical Trials and Biomedicine of West China Hospital of Sichuan University (IRB-2017-5). All eligible participates have to provide their signed informed consent prior to enrollment. The trial results will be disseminated through scientific journals or presentation at scientific conferences.

\section{Consent for publication}

Not applicable.

\section{Competing interests}

The authors declare that they have no competing interests.

\section{Publisher's Note}

Springer Nature remains neutral with regard to jurisdictional claims in published maps and institutional affiliations.

Received: 28 October 2018 Accepted: 13 March 2019

Published online: 29 March 2019

\section{References}

1. Grief SN. Upper respiratory infections. Prim Care. 2013;40:757-70.

2. Passioti M, Maggina P, Megremis S, Papadopoulos NG. The common cold: potential for future prevention or cure. Curr Allergy Asthma Rep. 2014;14:413.

3. Heikkinen T, Järvinen A. The common cold. Lancet. 2003:361:51-9.

4. Roelen CA, Koopmans PC, Notenbomer A, Groothoff JW. Job satisfaction and short sickness absence due to the common cold. Work (Reading, Mass). 2011:39:305-13.

5. Hsiao CJ, Cherry DK, Beatty PC, Rechtsteiner EA. National Ambulatory Medical Care Survey: 2007 summary. Natl Health Stat Rep. 2010:27:1-32.

6. Walter MJ, Castro M, Kunselman SJ, Chinchilli VM, Reno M, Ramkumar TP, et al. Predicting worsening asthma control following the common cold. Eur Respir J. 2008;32:1548-54.

7. Nahas R, Balla A. Complementary and alternative medicine for prevention and treatment of the common cold. Can Fam Physician. 2011;57:31-6.

8. Karsch-Volk M, Barrett B, Kiefer D, Bauer R, Ardjomand-Woelkart K, Linde K. Echinacea for preventing and treating the common cold. Cochrane Database Syst Rev. 2014;2:Cd000530.

9. Hemila H, Chalker E. Vitamin C for preventing and treating the common cold. Cochrane Database Syst Rev. 2013;1:Cd000980.

10. Vuichard GD, Dao D, Gysin CM, Lytvyn L, Loeb M. Effect of vitamin D3 supplementation on respiratory tract infections in healthy individuals: a systematic review and meta-analysis of randomized controlled trials. PLoS One. 2016;11:e0162996.

11. Hao Q, Dong BR, Wu T. Probiotics for preventing acute upper respiratory tract infections. Cochrane Database Syst Rev. 2015;2:Cd006895.
12. Seida JK, Durec T, Kuhle S. North American (Panax quinquefolius) and Asian ginseng (Panax ginseng) preparations for prevention of the common cold in healthy adults: a systematic review. Evid Based Complement Alternat Med. 2011;2011:282151.

13. Wu T, Zhang J, Qiu Y, Xie L, Liu GJ. Chinese medicinal herbs for the common cold. Cochrane Database Syst Rev. 2007;1:Cd004782

14. Li G, Cai L, Jiang H, Dong S, Fan T, Liu W, et al. Compound formulas of traditional Chinese medicine for the common cold: systematic review of randomized, placebo-controlled trials. Altern Ther Health Med. 2015;21:48-57.

15. Liu JB, Yao H, Xiao H. The research development in molecular epidemiology of humoral in Uygur medicine. J Xinjiang Med Univ. 2014;37:2.

16. Upur H, Yusup A, Baudrimont I, Umar A, Berke B, Yimit D, et al. Inhibition of cell growth and cellular protein, DNA and RNA synthesis in human hepatoma (HepG2) cells by ethanol extract of abnormal Savda Munziq of traditional Uighur medicine. Evid Based Complement Alternat Med. 2011;2011:251424.

17. Abuduwaili A, Silafu A, Wang P-S, Wubulihasimu YM, Muhamaiti W, et al. Clinical research guidelines for treatment of common cold with new Uyghur medicine (draft). Chin J Inf. 2017;24:1-4.

18. Ge JB, Xu YJ. Internal medicine. The 8th edition. Beijing, China: People's Medical Publishing House Press. 2013;2:13-14.

19. Wu F, Wang YJ, Hong YL, Feng Y, Ruan KF. Progress in preparation and clinical application of traditional Chinese medicine placebo. Chin J New Drugs. 2012;21:2161-4

20. China academy of Chinese medical sciences. Evidence-based guidelines of clinical practice in Chinese medicine (internal medicine). China: Traditional Chinese Medicine Press. 2011;311-328.

21. Cohen J. Statistical power analysis for the behavioral sciences. Technometrics. 1988:31:499-500.

22. Desu MM, Raghavarao D. Sample Size Methodology. Boston: Academic Press. 1990:35:125-131.

23. Kirk RE. Experimental design: procedures for the behavioral sciences. Belmont, CA: Brooks/Cole Publishing Company. 1968;26:52384-52392.

24. Ma ZQ, Hu H, He TT, Guo H, Zhang MY, Chen MW, et al. An assessment of traditional Uighur medicine in current Xinjiang region (China). Afr J Tradit Complement Altern Med. 2014;11:301-14.

\section{Ready to submit your research? Choose BMC and benefit from:}

- fast, convenient online submission

- thorough peer review by experienced researchers in your field

- rapid publication on acceptance

- support for research data, including large and complex data types

- gold Open Access which fosters wider collaboration and increased citations

- maximum visibility for your research: over $100 \mathrm{M}$ website views per year

At $\mathrm{BMC}$, research is always in progress.

Learn more biomedcentral.com/submissions 